\title{
ON FIXED POINTS OF NONEXPANSIVE SET-VALUED MAPPINGS
}

\author{
R. K. BOSE AND R. N. MUKHERJEE
}

\begin{abstract}
A theorem is proved concerning the existence of fixed points of nonexpansive set-valued mappings. This generalizes a result of Dotson, Jr. [1].
\end{abstract}

This note deals with the existence of fixed points of nonexpansive setvalued mappings from a compact subset of a complete metric space into itself. This result generalizes a theorem of Dotson, Jr. [1]

Definition. Let $(X, d)$ be a complete metric space, and let $S$ be a subset of $X$. We denote by $2^{S}$ the set of all compact subsets of $S$. Let $D(x, A)$ denote the ordinary distance between $x \in X$ and $A \in 2^{S}$. Let $F=\left\{f_{A}\right\}_{A \in 2^{s}}$ be a family of functions from $[0,1]$ into $2^{S}$ with the property that for each $A \in 2^{S}$, $f_{A}(1)=A$. Such a family is said to be contractive if there exists a function $\phi$ : $(0,1) \rightarrow(0,1)$ such that for all $A$ and $B$ in $2^{S}$ and for all $t \in(0,1)$ we have

$$
H\left(f_{A}(t), f_{B}(t)\right) \leqslant \phi(t) H(A, B)
$$

where $H$ is the Hausdorff metric. Such a family is said to be jointly continuous if $f_{A}(t) \rightarrow f_{A_{0}}\left(t_{0}\right)$ in $2^{S}$ whenever $t \rightarrow t_{0}$ in $[0,1]$ and $A \rightarrow A_{0}$ in $2^{S}$.

THEOREM. Let $S$ be a compact subset of a complete metric space. Suppose there exists a contractive, jointly continuous family $F$ of functions associated with $S$. Then any nonexpansive multi-valued mapping $T$ of $S$ into $2^{S}$ has a fixed point in $S$.

Proof. For each $n=1,2,3, \ldots$ let $r_{n}=n /(n+1)$, and let $T_{n}: S \rightarrow 2^{S}$ defined as $T_{n} x=f_{T x}\left(r_{n}\right)$ for all $x \in S$. $T_{n}$ is a well-defined map from $S$ into $2^{S}$ for each $n$. Also, for each $n$, and for all $x, y \in S$, we have

$$
\begin{aligned}
H\left(T_{n} x, T_{n} y\right) & =H\left(f_{T x}\left(r_{n}\right), f_{T y}\left(r_{n}\right)\right) \\
& \leqslant \phi\left(r_{n}\right) H(T x, T y) \leqslant \phi\left(r_{n}\right) d(x, y) .
\end{aligned}
$$

Hence, for each $n, T_{n}$ is a multi-valued contraction mapping from $S$ into $2^{S}$. Then by a theorem of Nadler, Jr. [2] there exist $x_{n} \in S$ such that $x_{n} \in T_{n} x_{n}$. Since $S$ is compact, there is a subsequence $\left\{x_{n_{j}}\right\}$ in $S$ of $\left\{x_{n}\right\}$ converging to $x_{0}$ in $S$. Also,

$$
x_{n_{j}} \in T_{n_{j}}\left(x_{n_{j}}\right)=f_{T x_{n_{j}}}\left(r_{n j}\right) \rightarrow_{j \rightarrow \infty} f_{T x_{0}}(1)=T x_{0},
$$

Received by the editors April 25, 1977 and, in revised form, November 18, 1977. AMS (MOS) subject classifications (1970). Primary 47H10; Secondary 47H99.

Key words and phrases. Nonexpansive set-valued mappings, fixed points. 
as $T x_{n_{j}} \rightarrow T x_{0}$ and $r_{n_{j}} \rightarrow 1$ (by joint continuity). Since $x_{n_{j}} \in T_{n_{j}}\left(x_{n_{j}}\right)$ for each $j$, it follows that $x_{0} \in T x_{0}$. Because

$$
\begin{aligned}
D\left(x_{0}, T x_{0}\right) & <d\left(x_{0}, x_{n_{j}}\right)+D\left(x_{n_{j}}, T x_{0}\right) \\
& \leqslant d\left(x_{0}, x_{n_{j}}\right)+H\left(T_{n_{j}} x_{n_{j}}, T x_{0}\right) .
\end{aligned}
$$

Hence $D\left(x_{0}, T x_{0}\right)=0$. As $T x_{0}$ is closed, $x_{0} \in T x_{0}$.

REMARK. Dotson, Jr. [1] has proved the theorem in a Banach space setting where the nonexpansive map is single-valued. However vector space structure of the space is not needed in the proof.

\section{REFERENCES}

1. W. G. Dotson, Jr., On fixed points of nonexpansive mappings in nonconvex sets, Proc. Amer. Math. Soc. 38 (1973), 155-156.

2. S. B. Nadler, Jr., Multi-valued contraction mappings, Pacific J. Math. 30 (1969), 473-488.

Mathematics Group, Birla Institute of Technology and Science, Pilani, Rnjasthan, INDIA 333031 